\title{
Factores asociados a mayor débito de drenaje tras linfadenectomía axilar por cáncer de mama*
}

\author{
Drs. JAIME RUIZ-TOVAR P. ${ }^{1}$, PILAR CANSADO M. ${ }^{1}$, MARÍA AMPARO GÓMEZ C. ${ }^{1}$, \\ MONTSERRAT PÉREZ S. ${ }^{1}$, LOREA ZUBIAGA T. ${ }^{1}$, MARÍA DIE TZ. ${ }^{1}$, RAFAEL CALPENA R. ${ }^{1}$ \\ 1 Unidad de Patología Mamaria, Servicio de Cirugía General y del Aparato Digestivo. \\ Hospital General Universitario de Elche, Alicante. \\ España.
}

\begin{abstract}
\section{Factors associated with greater debit drainage after axillary lymphadenectomy} for breast cancer
\end{abstract}

Introduction: Axillary lymph node dissection (ALND) is still a usual procedure in the treatment of breast cancer. A drain is normally placed in the surgical bed and maintained several days until obtaining a daily discharge of 30-50 ml, in order to reduce the appearance of seroma. The aim of this study was to analyze possible factors associated with an increased drainage volume. Patients and Methods: A retrospective study of all the patients undergoing ALND for breasts cancer at our institution between 2011 and 2012 was performed. Results: 40 females were included. There were no complications or mortality. Median hospital stay was 2 days. Median day of drainage removal was the 5th postoperative day. Mean total drainage volume was $298.9 \pm 240.1 \mathrm{ml}$. High blood pressure associated with an increased drainage volume (550 ml vs $217.5 \mathrm{ml}$; $\mathrm{p}=0.001)$ and a later removal $\left(8^{\text {th }} \mathrm{vs} 4^{\text {th }}\right.$ day; $\left.\mathrm{p}=0.002\right)$. Similar happened with age $>65$ years $(420.8 \mathrm{ml}$ vs $24.6 \mathrm{ml} ; \mathrm{p}=0.003)$ and $\left(7\right.$ th vs $5^{\text {th }}$ day; $\left.\mathrm{p}=0.009\right)$. The number of metastatic lymph nodes showed a direct correlation with total drainage volume (Pearson 0.503; $\mathrm{p}=0.017$ ) and removal day (Spearman 0.563; $\mathrm{p}=0.006)$, similar to the primary tumour size: Total drainage volume (Pearson $0.447 ; \mathrm{p}=0.042$ ) and removal day (Spearman 0.556; $\mathrm{p}=0.009$ ). Conclusion: Age over 65 years, high blood pressure, number of metastatic lymph nodes and primary tumour size associated with a higher drainage volume and time to remove the drain.

Key words: Lymphorrhea, drainage volume, axillary lymph node dissection.

\section{Resumen}

Introducción: La linfadenectomía axilar (LA) sigue siendo un procedimiento frecuente en el tratamiento del cáncer de mama. Habitualmente se coloca un drenaje en el hueco axilar, que se mantiene varios días, hasta que tiene un débito diario de 30-50 ml, con el fin de reducir la aparición de seroma. El objetivo del presente estudio es analizar posibles factores asociados a un mayor volumen de drenaje. Pacientes $\mathbf{y}$

*Recibido el 28 de marzo de 2013 y aceptado para publicación el 2 de mayo de 2013.

Los autores no refieren conflictos de interés.

Correspondencia: Dr. Jaime Ruiz-Tovar P. Fotógrafo Francisco Cano, 113, Bungalow 41. 03540-Alicante, España. jruiztovar@gmail.com 
Métodos: Realizamos un estudio retrospectivo de todas las pacientes intervenidas de LA por cáncer de mama en nuestro centro durante los años 2011 y 2012. Resultados: Incluimos 40 mujeres en el estudio. No hubo complicaciones ni mortalidad. La mediana de estancia hospitalaria fue de 2 días. El día mediano de retirada de drenaje fue el $5^{\circ}$ postoperatorio. El volumen medio total del drenaje fue 298,9 $\pm 240,1 \mathrm{ml}$. La hipertensión arterial se asocia a un mayor volumen de drenaje $(550 \mathrm{ml}$ vs $217,5 \mathrm{ml} ; \mathrm{p}=0,001)$ y a una retirada más tardía del mismo ( $8^{\circ}$ día vs $4^{\circ}$ día; $\left.p=0,002\right)$. Al igual ocurre con la edad $>65$ años $(420,8 \mathrm{ml}$ vs $24,6 \mathrm{ml} ; \mathrm{p}=0,003)$ y ( $7^{\circ}$ vs $5^{\circ}$ día; $\left.\mathrm{p}=0,009\right)$. El número de ganglios metastásicos también se correlaciona con el volumen total de drenaje (Coeficiente de correlación de Pearson 0,503; $p=0,017$ ) y con el día de retirada (Spearman 0,563; $\mathrm{p}=0,006$ ), lo mismo que el tamaño del tumor primario: volumen total de drenaje (Pearson 0,$447 ; \mathrm{p}=0,042$ ) y día de retirada (Spearman 0,556; $\mathrm{p}=0,009$ ). Conclusión: La edad mayor de 65 años, la hipertensión arterial, el número de ganglios metastásicos y el tamaño del tumor primario se asocian con mayor volumen por el drenaje axilar y días necesarios hasta la retirada.

Palabras clave: Linforrea, débito drenaje, linfadenectomía axilar.

\section{Introducción}

La linfadenectomía axilar (LA) sigue siendo un procedimiento habitual en el tratamiento del cáncer de mama, a pesar de que en los últimos años el número de procedimientos realizados ha disminuido significativamente gracias a la implantación de la biopsia selectiva del ganglio centinela $(\mathrm{BSCG})^{1}$. En la cirugía de la LA se coloca un drenaje en el hueco axilar, que se mantiene varios días con el fin de reducir la aparición de seroma y posterior sobreinfección a absceso axilar ${ }^{2}$. No obstante, el mantenimiento de este drenaje implica un aumento de la estancia hospitalaria hasta su retirada ${ }^{3}$ o que el paciente sea dado de alta antes con el drenaje, lo que disminuye el confort y aumenta el impacto psicológico de la enfermedad ${ }^{2,4}$.

Se ha demostrado que el volumen de débito del drenaje axilar y la aparición de seroma, si el drenaje es retirado de forma precoz, son independientes de la técnica quirúrgica realizada, de los instrumentos de disección y sellado utilizados ${ }^{5-8} \mathrm{o}$ de la adición de sellantes de fibrina ${ }^{9}$.

El objetivo del presente estudio es analizar posibles factores asociados a un mayor volumen de drenaje y analizar si alguno de ellos pudiera ser modificable, de cara a poder retirar el drenaje precozmente y sin riesgo de desarrollar seroma, lo que mejoraría la calidad de vida de estas pacientes.

\section{Pacientes y Métodos}

Realizamos un estudio retrospectivo de todas las pacientes intervenidas de LA por cáncer de mama en el Servicio de Cirugía General del Hospital General Universitario de Elche durante los años 2011 y 2012.

Los criterios de inclusión fueron mujeres con diagnóstico de cáncer de mama y sometidas a una LA de los niveles I y II de Berg por metástasis axilares diagnosticadas de forma preoperatoria mediante biopsia con aguja gruesa o con evidencia de metástasis en el estudio intraoperatorio o diferido de la BSGC. Fueron excluidas aquellas pacientes sometidas a mastectomía radical modificada.

Se empleó profilaxis antibiótica con Amoxicilina/ Ácido Clavulánico $2 \mathrm{~g}$ iv, a dosis única preoperatoria, $30 \mathrm{~min}$ antes de la incisión.

La disección axilar se realizó mediante bisturí armónico (Ultracision, Ethicon Endosurgery, Johnson and Johnson, Cincinnati, OH, USA). Una vez finalizada la intervención y previamente al cierre de la piel, se colocó un drenaje tipo Redon, que se conectó a un sistema de baja presión. Se utilizó una sutura de puntos simples de Poliglactina 3/0 para aproximar el tejido celular subcutáneo y una sutura intradérmica de Poliglitona 3/0 para cerrar la piel. Por último, se aplicó un vendaje compresivo sobre el hueco axilar.

Las pacientes fueron dadas de alta con el drenaje, solicitándoles que cuantificasen diariamente el volumen. El criterio para retirada del drenaje fue cuando el débito era menor de $30 \mathrm{ml} /$ día.

Se realizó un seguimiento diario en Consultas Externas hasta la retirada del drenaje y posteriormente de forma semanal hasta cumplirse el primer mes postoperatorio para valorar la aparición de seroma o infección de herida quirúrgica.

\section{Variables}

Las variables clínicas analizadas fueron edad, índice de masa corporal (IMC), comorbilidades (diabetes mellitus, hipertensión, cardiopatía, neumopatía, nefropatía, toma de medicación antiagregante o anticoagulante), BSGC realizada un acto quirúrgico previo, complicaciones (infección de herida, seroma), mortalidad, estancia hospitalaria, volumen total del drenaje hasta su retirada y día de retirada. Las variables histopatológicas evaluadas fueron número de ganglios aislados en la LA, número de ganglios positivos y tamaño del tumor primario. 


\section{Análisis estadístico}

Se realizó utilizando el programa SPSS 19.0 para Windows. Las variables cuantitativas que seguían una distribución normal se definieron por media y desviación típica, mientras que en las que no presentaban distribución gaussiana se emplearon mediana e intervalo. Las variables discretas fueron definidas por número de casos y porcentaje.

La comparación entre variables se realizó mediante los tests t de Student y correlación de Pearson, utilizando las pruebas no paramétricas equivalentes, cuando alguna variable no seguía distribución normal. La comparación entre variables discretas se realizó mediante el test de $\chi^{2}$, con la corrección de Fisher en caso necesario. La magnitud de la asociación fue cuantificada mediante Riesgo Relativo. Se consideraron significativos aquellos valores de $\mathrm{p}<0,05$.

\section{Resultados}

Se trataron un total de 282 pacientes con cáncer de mama entre los años 2011 y 2012. De ellas, 40 pacientes fueron sometidas a linfadenectomía axilar, todas ellas mujeres, con una edad media de $54,8 \pm 13,7$ años. Presentaban un IMC medio de $27,2 \pm 4,6 \mathrm{~kg} / \mathrm{m}^{2}$. Cuatro mujeres $(10 \%)$ presentaban diabetes mellitus tipo 2, 8 (20\%) hipertensión arterial y $2(5 \%)$ cardiopatía isquémica. Cuatro (10\%) mujeres tomaban ácido acetilsalicílico como medicación habitual. Todas las pacientes hipertensas estaban en tratamiento con inhibidores del enzima convertidor de angiotensina. A pesar de ello, su tensión arterial sistólica preoperatoria media estaba por encima del rango de la normalidad $(139,2 \mathrm{mmHg}$; rango $170-130 \mathrm{mmHg}$ ) y era significativamente mayor que en los pacientes sin diagnóstico previo de hipertensión arterial (media 121,4 mmHg; rango 141-112 mmHg) $(\mathrm{p}=0,042)$.

En 29 pacientes $(72,5 \%)$ la LA se realizó tras confirmación histológica de la presencia de metástasis en el estudio intraoperatorio de la BSGC. En 4 mujeres (10\%) la LA se efectuó en un segundo acto quirúrgico por confirmación de metástasis en el estudio diferido del ganglio centinela, cuyo análisis intraoperatorio fue negativo para malignidad. En 7 pacientes $(17,5 \%)$ la LA se realizó directamente, ante el diagnóstico preoperatorio de metástasis axilar mediante biopsia con aguja gruesa.

No hubo complicaciones (seroma o infección de herida, antes ni después de la retirada del drenaje), ni mortalidad. La mediana de estancia hospitalaria fue de 2 días (intervalo 1-3 días). El día mediano de retirada de drenaje fue el $5^{\circ}$ postoperatorio (intervalo 3-16 días). El volumen medio total del drenaje fue
$298,9 \pm 240,1 \mathrm{ml}$; mediana $177,5 \mathrm{ml}$ (intervalo 50$820 \mathrm{ml})$.

El número medio de ganglios linfáticos aislados fue $21,3 \pm 6,1$ y de ganglios metastásicos $3,4 \pm 2$. El tamaño medio del tumor primario fue $2,6 \pm 1,4 \mathrm{~cm}$.

\section{Factores asociados con el volumen total de dre- naje y día de retirada del mismo}

La hipertensión arterial se asocia a un mayor volumen de drenaje $(550 \mathrm{ml}$ frente a $217,5 \mathrm{ml}$ en pacientes no hipertensos; $p=0,001)$ y a una retirada más tardía del mismo ( $8^{\circ}$ día frente a $4^{\circ}$ día; $\mathrm{p}=0,002)$.

La edad se correlaciona con el volumen total de drenaje (Coeficiente de correlación de Pearson $0,276 ; p=0,008)$, pero no con el día de retirada del drenaje, aunque muestra una tendencia a la misma $(\mathrm{p}=0,08)$. Estableciendo un punto de corte de máxima discriminación en 65 años, apreciamos que los pacientes mayores de esta edad presentan un volumen total mayor $(420,8 \mathrm{ml}$ frente a $24,6 \mathrm{ml}$; $\mathrm{p}=0,003)$ y también un día de retirada de drenaje más tardío $\left(7^{\circ}\right.$ frente a $5^{\circ}$ día; $\left.\mathrm{p}=0,009\right)$.

El número de ganglios metastásicos también se correlaciona con el volumen total de drenaje (Coeficiente de correlación de Pearson 0,503; $\mathrm{p}=0,017)$ y con el día de retirada del mismo (Coeficiente de correlación de Spearman 0,563; $p=0,006$ ).

Igualmente, el tamaño del tumor primario, presenta una correlación directa con el volumen total de drenaje (Coeficiente de correlación de Pearson $0,447 ; p=0,042)$ y con el día de retirada del mismo (Coeficiente de correlación de Spearman 0,556; $\mathrm{p}=0,009)$.

Todos los factores asociados con el volumen total de drenaje y el día de retirada del mismo se resumen en las Tablas 1 y 2.

En el análisis multivariado, todas las variables mostraron ser factores pronósticos independientes.

El resto de variables analizadas no mostraron correlación con el volumen total de drenaje ni con el día de retirada del mismo.

\section{Discusión}

Una de las complicaciones más frecuentes tras la LA es la aparición de un linfocele o seroma, a consecuencia de la sección de canalículos linfáticos durante la disección axilar. Este depósito de linfa en el hueco axilar puede llegar a sobreinfectarse y originar un absceso axilar. Para evitar este acúmulo de linfa postoperatorio, el único método que ha demostrado ser eficaz hasta la fecha es la colocación de un drenaje en el lecho quirúrgico, que exteriorice el líquido y evite su acumulación ${ }^{10}$. Este drenaje debe 
Tabla 1. Factores asociados con el día de retirada del drenaje

\begin{tabular}{|llcc|}
\hline \multicolumn{1}{|c|}{} & \multicolumn{1}{c|}{ Valores } & Coeficiente de correlación de Pearson & p \\
\hline Hipertensión arterial (HTA) & HTA: $8^{\circ}$ día & & 0,002 \\
& No HTA: $4^{\circ}$ día & & 0,009 \\
Edad & $>65$ años: $7^{\circ}$ día & & 0,017 \\
n de ganglios metastásicos & $<65$ años: $5^{\circ}$ día & 0,503 & 0,042 \\
Tamaño del tumor primario & & 0,447 & \\
\hline
\end{tabular}

Tabla 2. Factores asociados con el volumen total de drenaje

\begin{tabular}{|llcc|}
\hline \multicolumn{1}{|c|}{ Valores } & Coeficiente de correlación de Spearman & p \\
Hipertensión arterial (HTA) & HTA: $550 \mathrm{ml}$ & & 0,001 \\
& No HTA: $217,5 \mathrm{ml}$ & & 0,003 \\
Edad & $>65$ años: $420,8 \mathrm{ml}$ & & 0,006 \\
n de ganglios metastásicos & $<65$ años: $24,6 \mathrm{ml}$ & 0,563 & 0,009 \\
\hline Tamaño del tumor primario & & 0,556 & 0 \\
\hline
\end{tabular}

ser mantenido en el tiempo hasta que su débito prácticamente desaparezca, lo que implica o una mayor estancia hospitalaria o la incomodidad del manejo del drenaje de forma ambulatoria ${ }^{2-4}$.

Durante la disección axilar no sólo se seccionan los canalículos linfáticos, que originan la extravasación de la linfa al espacio intersticial, sino que la propia agresión quirúrgica desencadena una respuesta inflamatoria. Las células inflamatorias (neutrófilos, linfocitos y macrófagos), abandonan la circulación sanguínea y migran hacia el foco inflamatorio a través de la circulación linfática, que es un camino excelente para alcanzarlo. Acompañando a las células inflamatorias, también se extravasan agua y proteínas, a consecuencia del aumento de la permeabilidad vascular en respuesta a las citoquinas y otros mediadores de la respuesta local a la agresión. Esta agua también fluye por la red linfática y se extravasa al espacio intersticial, condicionando el edema de los tejidos inflamados ${ }^{11,12}$. Todo ello, añadido a la ruptura quirúrgica de los canalículos, contribuye a la linforrea típica tras LA, mayor de la esperada simplemente por un proceso inflamatorio.

Se han identificado varios factores asociados a un mayor volumen de drenaje o incluso a la aparición de seroma tras la retirada del mismo, sobre todo cuando éste era retirado de forma precoz ${ }^{13}$. Entre ellos destaca la edad, la obesidad, el hábito tabáquico, el tratamiento neoadyuvante con radioquimioterapia, el número de ganglios extirpados y el número de ganglios metastásicos ${ }^{14-16}$. En nuestro estudio no había ningún paciente incluido que recibiera tratamiento neoadyuvante y el hábito tabáquico no fue recogido de forma rutinaria en la historia clínica. El IMC no se correlacionó con el volumen de drenaje ni el día de retirada del mismo, probablemente por el pequeño tamaño muestral. En lo referente a la edad, observamos una correlación directa con el volumen de drenaje. Establecimos un punto de corte de máxima discriminación en 65 años. En ancianos se ha observado una disfunción endotelial que acaba desembocando en una mayor permeabilidad, afectando tanto al endotelio vascular como al linfático. El daño endotelial permite la salida de moléculas de mayor tamaño, fundamentalmente proteínas, que al aumentar la presión oncótica extravascular, arrastra la salida de agua. Esta extravasación de agua y proteínas es recogida por vía linfática, aumentando el flujo linfático y justificando, por tanto, una mayor linforrea en ancianos. El daño endotelial parece producirse por un aumento en la formación de radicales libres de oxígeno y una reducción en la producción de agentes antioxidantes. Esto contribuye a una disminución de liberación de óxido nítrico por parte de las células endoteliales, que es la sustancia encargada de regular la relajación del músculo liso vascular y la permeabilidad capilar ${ }^{17,18}$.

En nuestro estudio también hemos observado un aumento del volumen de drenaje axilar en pacientes hipertensos. Este factor ya ha sido descrito 
como un factor favorable de linforrea por algunos autores $^{10,15,16}$, aunque no confirmado por otros ${ }^{14}$. El aumento de la presión sanguínea también favorece la extravasación de agua de la circulación sanguínea al espacio extracelular. La hipertensión arterial esencial es consecuencia de la arterioesclerosis en una importante proporción de casos. A la génesis de la arteriosclerosis también contribuye la disminución de síntesis de óxido nítrico, que no contrarresta el efecto vasoconstrictor de la angiotensina II, favoreciendo así la hipertensión arterial ${ }^{19}$. La hipertensión arterial es el único factor modificable de todos los que hemos demostrado en nuestro estudio, por lo que es recomendable un control estricto de la misma mediante un incremento de la medicación antihipertensiva, tanto durante el preoperatorio como en el postoperatorio.

El volumen de drenaje también se ha asociado con el número de ganglios metastásicos, lo que a ha sido descrito por otros autores ${ }^{14}$. Kett demostró que las células tumorales en los ganglios linfáticos axilares obstruyen el flujo linfático normal, aumenta la presión dentro de los vasos y capilares linfáticos y ante la presencia de soluciones de continuidad en los mismos, aumenta la salida la linfa y crea mayor linforrea ${ }^{20}$.

Hasta la fecha no se ha descrito la asociación entre el tamaño del tumor primario y la linforrea. A pesar de que está ampliamente demostrado que el tamaño tumoral se asocia con el compromiso $\operatorname{axilar}^{21}$, en nuestra serie el tamaño tumoral no se asociaba con el número de ganglios metastásicos, posiblemente debido al pequeño tamaño muestral. No obstante, en el análisis multivariado se demostró que el tamaño tumoral era un factor pronóstico independiente de mayor débito por el drenaje.

\section{Conclusión}

La edad mayor de 65 años, la hipertensión arterial, el número de ganglios linfáticos metastásicos y el tamaño del tumor primario son factores predictores de mayor volumen por el drenaje axilar y por tanto, son necesarios mayor número de días transcurridos hasta poder retirar el drenaje. La hipertensión arterial es el único factor modificable, por lo que se recomienda un estricto control de la misma antes y después de la intervención.

\section{Referencias}

1.- Rutgers EJ, Meijnen P, Bonnefoi H. European Organization for Research and Treatment of Cancer Breast Cancer Group. Clinical trials update of the European Organization for Research and Treatment of Cancer
Breast Cancer Group. Breast Cancer Res. 2004;6:165-9.

2.- Andeweg CS, Schriek MJ, Heisterkamp J, Roukema JA. Seroma formation in two cohorts after axillary lymph node dissection in breast cancer surgery: does timing of drain removal matter? Breast J. 2011;17:359-64.

3.- He XD, Guo ZH, Tian JH, Yang KH, Xie XD. Whether drainage should be used after surgery for breast cancer? A systematic review of randomized controlled trials. Med Oncol. 2011;28:22-30.

4.- Del Bianco P, Zavagno G, Burelli P, Scalco G, Barutta L, Carraro P, et al. Morbidity comparison of sentinel lymph node biopsy versus conventional axillary lymph node dissection for breast cancer patients: results of the sentinella-GIVOM Italian randomised clinical trial. Eur J Surg Oncol. 2008;34:508-13.

5.- Iovino F, Auriemma PP, Ferraraccio F, Antoniol G, Barbarisi A. Preventing seroma formation after axillary dissection for breast cancer: a randomized clinical trial. Am J Surg. 2012;203:708-14.

6.- Sanguinetti A, Docimo G, Ragusa M, Calzolari F, D’Ajello F, Ruggiero R, et al. Ultrasound scissors versus electrocautery in axillary dissection: our experience. G Chir. 2010;31:151-3.

7.- Cortadellas T, Córdoba O, Espinosa-Bravo M, Mendoza-Santin C, Rodríguez-Fernández J, Esgueva A, et al. Electrothermal bipolar vessel sealing system in axillary dissection: a prospective randomized clinical study. Int J Surg. 2011;9:636-40.

8.- Manouras A, Markogiannakis H, Genetzakis M, Filippakis GM, Lagoudianakis EE, Kafiri G, et al. Modified radical mastectomy with axillary dissection using the electrothermal bipolar vessel sealing system. Arch Surg. 2008;143:575-80.

9.- van Bemmel AJ, van de Velde CJ, Schmitz RF, Liefers GJ. Prevention of seroma formation after axillary dissection in breast cancer: a systematic review. Eur J Surg Oncol. 2011;37:829-35.

10.- Douay N, Akerman G, Clement D, Malartic C, Morel O, Barranger E. Seroma after axillary lymph node dissection in breats cancer. Gynecol Obstet Fertil. 2008;36:130-5.

11.- Cotran RS. Robbins Pathologic Basis of Disease. Philadelphia: WB Saunders Company, 1998.

12.- Balibrea Cantero JL. Patología Quirúrgica. Madrid: Marban, 2003.

13.- Droeser RA, Frey DM, Oertli D, Kopelman D, BaasVrancken MJ, Giuliano AE, et al. Volume controlled vs no/short-term drainage after axillary lymph node dissection in breast cáncer surgery: a meta-analysis. Breast 2009;18:109-14.

14.- Ozaslan C, Kuru B. Lymphedema after treatment of breast cancer. Am J Surg. 2004;187:69-72.

15.- Xue DQ, Qian C, Yang L, Wang XF. Risk factors for surgical site infections after breast surgery: a systematic review and meta-analysis. Eur J Surg Oncol. 2012;38:375-81. 
16.- Akinci M, Cetin B, Aslan S, Kulacoglu H. Factors affecting seroma formation after mastectomy with full axillary dissection. Acta Chir Belg. 2009;109:481-3.

17.- Garaliene V. The main determinants of endothelial dysfunction. Medicina (Kaunas) 2006;42:362-9.

18.- Starr ME, Ueda J, Yamamoto S, Evers BM, Saito H. The effects of aging on pulmonary oxidative damage, protein nitration, and extracellular superoxide dismutase down-regulation during systemic inflammation. Free Radic Biol Med. 2011;50:371-80.

19.- Dell'omo G, Penno G, Pucci L, Lucchesi D, Fotino C,
Del Prato S, et al. ACE gene insertion/deletion polymorphism modulates capillary permeability in hypertension. Clin Sci. (Lond) 2006;111:357-64.

20.- Kett K. Lymph drainage as prognostic factor in breast carcinoma. Langenbeckss Arch Chir Suppl Kongressbd. 1997;114:1219-21

21.- Robinson BD, Sica GL, Liu YF, Rohan TE, Gertler FB, Condeelis JS, et al. Tumor microenvironment of metastasis in human breast carcinoma: a potential prognostic marker linked to hematogenous dissemination. Clin Cancer Res. 2009;15:2433-41. 